\title{
DESIGN OF A 6 TeV MUON COLLIDER*
}

\author{
M-H. Wang ${ }^{\dagger}$, Y. Nosochkov, Y. Cai, SLAC, Menlo Park, CA 94025, USA \\ M. Palmer, FNAL, Batavia, IL 60510, USA
}

\section{Abstract}

A design of a muon collider ring with the center of mass energy of $6 \mathrm{TeV}$ is presented. The ring circumference is about $6.3 \mathrm{~km}$, and the $\beta$ functions at collision point are $1 \mathrm{~cm}$ in both planes. The ring linear optics, the non-linear chromaticity correction scheme in the Interaction Region (IR), and the additional non-linear field orthogonal knobs are described. The IR magnet specifications are based on the maximum pole tip field of $20 \mathrm{~T}$ in dipoles and $15 \mathrm{~T}$ in quadrupoles. The results of the beam dynamics optimization for maximum dynamic aperture are presented.

\section{INTRODUCTION}

A muon collider is one of the potential candidates for a future energy frontier colliding machine. Considering requirements on energy, luminosity and wall power in operation, the preferred choice of the center of mass muon beam energy appears to be near $6 \mathrm{TeV}$ [1]. In order to obtain peak luminosity of $\geq 10^{34} \mathrm{~cm}^{-2} \mathrm{~s}^{-1}$ in the TeV range, a number of demanding requirements to the collider optics should be satisfied [2]. These include a low beta function $\left(\beta^{*}\right)$ at the Interaction Point (IP), small circumference for high revolution frequency, large dynamic aperture, and low momentum compaction factor for a short bunch length $\left(\sigma_{\mathrm{z}}<\beta^{*}\right)$. The requirements are arising from a short muon lifetime and relatively large values of transverse emittance and momentum spread that can be realistically achieved with ionization cooling. They also come from the limitations on the maximum magnetic fields as well as the necessity to protect superconducting (SC) magnets and collider detectors from muon decay products. Another complication is the "hot spots" of radiation which can be induced by neutrinos from muon decay in straight sections [3]. As a result, straight sections without bending field must be very short. More detailed discussion of these requirements can be found in Ref. [2].

\section{LINEAR OPTICS}

For a $6 \mathrm{TeV}$ muon collider ring, achieving a short circumference and strong focusing in the IR requires rather high magnetic field. In this design, we chose the circumference of $\approx 6.3 \mathrm{~km}$ (approximately the size of the Tevatron) and the maximum pole-tip field of $20 \mathrm{~T}$ in dipoles and $15 \mathrm{~T}$ in quadrupoles. We expect that future advances in SC magnet design will make achieving such a strong field possible. The designed ring lattice has a twofold symmetry (periodicity) and consists of two identical IRs and two arcs.

* Work supported by the US DOE No. DE-AC02-76SF00515 and HEP. $\dagger$ mhwang@slac.stanford.edu

\section{Interaction Region}

The Interaction Region is the most challenging part of a high luminosity collider due to the extremely high beta functions in the final focusing (FF) quadrupoles generating large non-linear chromaticity as well as creating high sensitivity to magnet errors. The designed optics of one half of the IR of the $6 \mathrm{TeV}$ machine is shown in Fig. 1, where the IP is on the left-hand side with $\beta^{*}=1 \mathrm{~cm}$ in $x$ and $y$ planes. The IP is followed by a $6 \mathrm{~m}$ free space and a FF quadrupole doublet where vertical beta function reaches an extremely high value of $134 \mathrm{~km}$. The remaining part of the IR is made of FODO-like lattice where most of the space between the quadrupoles is filled with dipole magnets to avoid hot spots of neutrino radiation. Due to the high beta functions, the FF quadrupoles generate very large non-linear chromaticity resulting in a non-linear chromatic tune shift and large energy dependent perturbation of beta functions. These effects must be locally compensated to avoid a severe degradation of the ring momentum acceptance.

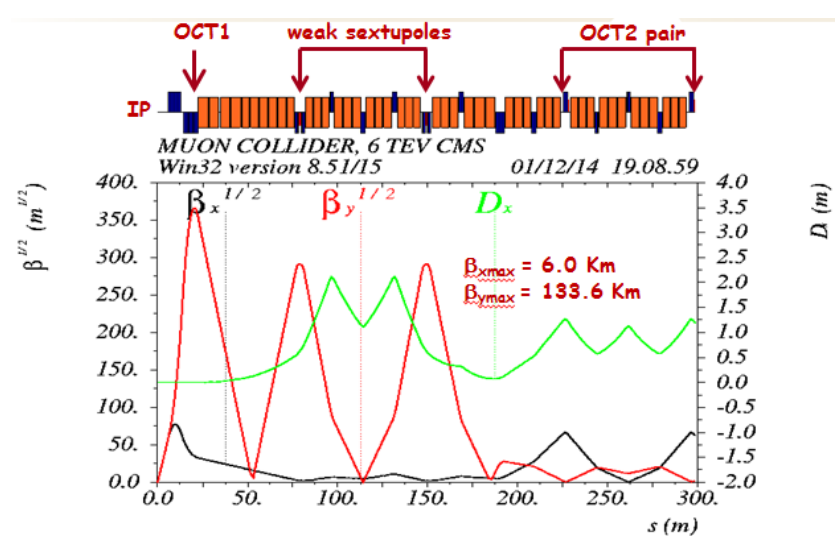

Figure 1: Linear optics of one half of the IR starting from IP. Two - I sextupole pairs ( $y$ and $x$ ) are placed at large $\beta_{\mathrm{y}}$ and $\beta_{\mathrm{x}}$ peaks for local correction of the FF quadrupole non-linear chromaticity. Positions of weak sextupoles and octupoles for additional non-linear correction are also shown.

The designed IR chromaticity correction scheme is based on two ( $x$ and $y$ ) non-interleaved pairs of sextupoles on each side of the IP, where the sextupoles in each pair are separated by $-\mathrm{I}$ transformation for cancellation of the $2^{\text {nd }}$ order sextupole geometric (amplitude dependent) aberrations. The sextupoles are placed near the high beta peaks $(x$ and $y$ ) to maximize their effective strengths. This correction scheme will be discussed in more detail later. At each end of the IR there is a dispersion suppressor FODO section followed by a $\sim 40 \mathrm{~m}$ of non-dispersive 
straight. The latter can be used for beam injection, placement of RF-cavities as well as geometric (harmonic) sextupoles or octupoles for additional non-linear compensation.

\section{Arcs}

The arc lattice of the $6 \mathrm{TeV}$ ring is based on the lattice design for the $1.5 \mathrm{TeV}$ muon collider (by Y. Alexahin [4]). The $6 \mathrm{TeV}$ arc cell has the same length as in the $1.5 \mathrm{TeV}$ design, but due to the longer circumference the bending angle per cell is reduced, and the number of cells per each arc is increased from 6 to 16 . Each cell contains three sextupole families for correction of linear chromaticity. Phase advance per arc cell is changed from the original $\mu_{\mathrm{x}} / \mu_{\mathrm{y}}=0.833 / 0.833[2 \pi]$ to $0.875 / 0.875$ [2 $\left.\pi\right]$. The latter provides a unit transformation per each half arc leading to local cancellation of most of the sextupole non-linear geometric aberrations. The magnet pole-tip field is increased with the higher energy, but is kept below $15 \mathrm{~T}$ in quadrupoles and $20 \mathrm{~T}$ in dipoles.

\section{CHROMATICITY CORRECTION}

Chromaticity of a collider with low $\beta^{*}$ is dominated by the FF quadrupoles. Due to the extremely high beta functions in these quadrupoles, they generate not only a significant part of linear chromaticity, but also are the main source of non-linear chromaticity such as a nonlinear chromatic tune shift and energy dependent perturbation of beta functions. These non-linear effects must be compensated to avoid the chromatic beta perturbation to propagating around the ring, thus further increasing the non-linear effects and severely limiting the ring momentum acceptance. This requires a dedicated local correction system using sextupoles in the IR. The included chromaticity correction scheme is based on two non-interleaved pairs of IR sextupoles (SIRY1-SIRY2 and SIRX1-SIRX2) for $y$ and $x$ correction on each side of IP, where the sextupoles in each pair are separated by $-\mathrm{I}$ transformation for cancellation of the $2^{\text {nd }}$ order sextupole geometric (amplitude dependent) aberrations. To provide an efficient correction of the FF chromaticity, the following conditions at the sextupoles are implemented: 1) sufficiently large dispersion and very large beta function to provide a required sextupole effect with a limited sextupole magnetic field; 2) large $\beta_{\mathrm{x}} / \beta_{\mathrm{y}}$ or $\beta_{\mathrm{y}} / \beta_{\mathrm{x}}$ ratio for orthogonal $x$ and $y$ correction; 3 ) $n \pi$ phase advance (in the correcting plane) between the FF quads and the sextupoles; 4) no other quadrupoles between the FF doublet and the first sextupole to avoid chromatic perturbation of phase advance between them. The sextupoles are placed at the double beta peaks $(y$ and $x$ ) in Fig. 1.

The remaining linear chromaticity of the machine (after the IR correction) can be canceled using three families of sextupoles in the periodic arc cells. These sextupoles are arranged in a way that cancels many of the sextupole driven non-linear effects locally in each arc. This is made possible by choosing an optimal cell phase advance of
$0.875 / 0.875[2 \pi]$. The resulting $+\mathrm{I}$ transformation in each half arc ( 8 periodic cells) makes the latter an almost exact fourth order geometric achromat $[5,6]$. The remaining non-vanishing effects of the arc sextupoles are the resonance driving term $2 v_{\mathrm{x}}-2 v_{\mathrm{y}}$ and five phase independent terms: two second order chromatic tune terms and three amplitude dependent tune terms.

The initial set-up of the IR and the arc sextupole strengths was performed in MAD [7] using the following method. First, the IR sextupole strengths were adjusted to locally cancel the $x$ and $y \mathrm{~W}$-functions [7] at the IP as shown in Fig. 2. This way, the chromatic variation of IP beta functions and, therefore, the IP beam size, are minimized. With this correction, the second order terms of the chromatic tune shift generated by the FF quadrupoles are compensated as well. As a second step, the remaining ring linear chromaticity is cancelled using the three sextupole families in the arcs. This two-step procedure can be used iteratively, if needed, to obtain simultaneous cancellation of the IP W-functions and the ring linear chromaticity. The effect of this compensation on beam dynamic aperture was verified in tracking simulations using LEGO [8] for machine without magnet errors. Fig. 3 shows the dynamic aperture at IP for on and off-momentum particles up to $\Delta \mathrm{p} / \mathrm{p}=0.4 \%$. One can see that the vertical dynamic aperture is rather poor (about $2 \sigma_{\mathrm{y}}$ ), and the momentum range is limited to $\Delta \mathrm{p} / \mathrm{p}=0.4 \%$. Further studies reveal that the vertical amplitude dependent tune shift and the horizontal chromatic tune shift are large. Therefore, additional non-linear compensation is required in order to reduce these effects. The goal of this compensation is to increase the $x$ and $y$ dynamic aperture to more than $5 \sigma$, where $\sigma=3 \mu \mathrm{m}$ (at IP) at $3 \mathrm{TeV}$ energy per beam, and increase the momentum range to at least $\pm 0.5 \%$.

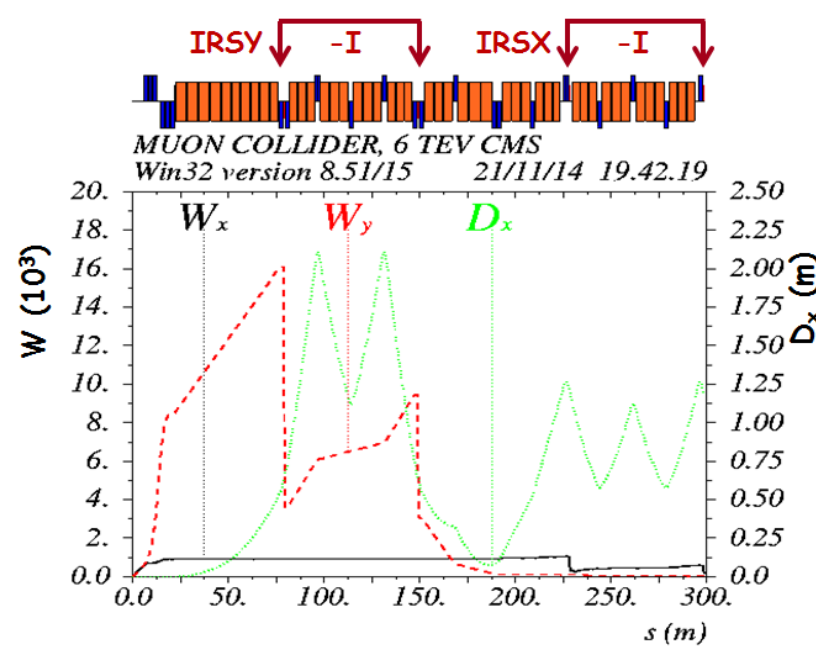

Figure 2: W-functions are locally cancelled at the IP ( $\mathrm{S}=$ 0 ) and outside of the IR using the two -I sextupole pairs. The remaining machine linear chromaticity is canceled using arc sextupoles. 


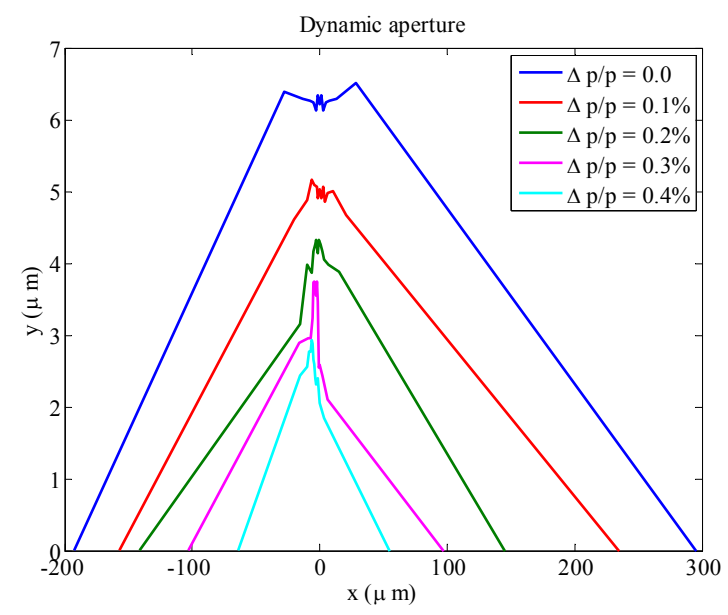

Figure 3: Dynamic aperture at IP for the ring with -I sextupole pairs in the IR and three sextupole families in the arcs. The vertical aperture is not sufficient, and the momentum range is below $0.5 \%$.

\section{FURTHER COMPENSATION OF NON-LINEAR EFFECTS}

Dynamic aperture simulations with the local IR chromaticity correction revealed that the vertical dynamic aperture is not sufficient due to the large vertical amplitude dependent tune shift. Secondly, the range of stable off-momentum motion is limited to $\Delta \mathrm{p} / \mathrm{p}=0.4 \%$ due to large horizontal chromatic tune shift. In order to improve the dynamic aperture, additional orthogonal nonlinear correction systems (knobs) were added in the IR. These include (per half-IR): 1) one octupole (OCT1) at a non-dispersive location with large vertical beta function to correct the vertical amplitude dependent tune shift; 2) a pair of weak sextupoles placed symmetrically around each main SIRY1 and SIRY2 sextupoles to compensate the effect of the finite sextupole length (the latter is amplified by the very high beta function) [9]; and 3) a $-\mathrm{I}$ pair of opposite polarity octupoles (OCT2) near the main SIRX1, SIRX2 sextupoles (where $\beta_{\mathrm{x}}$ and dispersion are large) to correct the $3^{\text {rd }}$ order horizontal chromatic tune shift. Positions of these sextupoles and octupoles in the IR are shown in Fig. 1. The optimized dynamic aperture after these corrections is shown in Fig. 4, where the integrated strengths of OCT1 and OCT2 octupoles are $\mathrm{K}_{3} \mathrm{~L}=0.0147$ $\mathrm{m}^{-3}$ and $\pm 14.5 \mathrm{~m}^{-3}\left(K_{3}=\frac{B^{\prime \prime \prime}}{6 B \rho}, \mathrm{L}\right.$ is the effective length), and the ratio of the weak sextupole strength to the main SIRY1/SIRY2 strength is -0.0595 . One can see that this correction improves the vertical aperture a factor of 4 relative to Fig. 3, and the momentum range is increased to $0.5 \%$. More detailed discussions of this compensation can be found in Ref. [10].

\section{SUMMARY}

The lattice design of a muon collider ring with $6 \mathrm{TeV}$ $\mathrm{CM}$ energy and circumference of $6.3 \mathrm{~km}$ is presented. The very high beta functions in the FF quadrupoles cause strong non-linear chromatic aberrations limiting the dynamic aperture. To minimize these effects, we include non-interleaved -I pairs of IR sextupoles for local chromaticity correction as well as additional pairs of IR weak sextupoles and two families of octupoles to compensate the $3^{\text {rd }}$ order horizontal chromatic tune shift, the non-linear effects of finite length of the IR sextupoles, and the vertical amplitude dependent tune shift. Additionally, the arc lattice is designed for local cancellation of sextupole driven resonance driving terms in each half-arc. Optimization of these non-linear corrections results in sufficient dynamic aperture within the momentum range of $\pm 0.5 \%$.

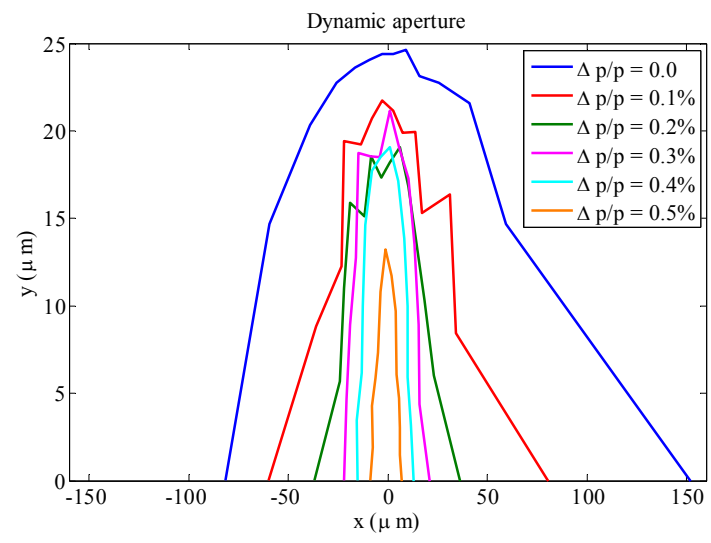

Figure 4: Dynamic aperture with the optimized IR nonlinear correction using OCT1 and OCT2 octupoles and the weak sextupoles.

\section{ACKNOWLEDGMENT}

Authors would like to thank Dr. Y. Alexahin for providing the lattice for $1.5 \mathrm{TeV} \mathrm{CM}$ muon collider ring and the reference materials.

\section{REFERENCES}

[1] E. Eichten, MAP Spring Meeting, FNAL (2014).

[2] Y. Alexahin et al., Proc. of PAC'09, TH6PFP051, Vancouver, BC, Canada (2009).

[3] N.V. Mokhov, A.V. Van Ginneken, J. Nucl. Sci. Techn. 37:sup1, 172-179 (2000).

[4] Y. Alexahin, private communication.

[5] Y. Cai et al., Phys. Rev. ST Accel. Beams 15, 054002 (2012).

[6] K. L. Brown and R. V. Servranckx, Nucl. Instrum. Methods in Phys. Res. A 258, 480 (1987).

[7] H. Grote and F.C. Iselin, "The MAD Program User's Reference Manual," CERN/SL/90-13 (AP).

[8] Y. Cai, M. Donald, I. Irwin, Y. Yan, SLAC-PUB7642 (1997).

[9] Y. Cai, Future Circular Collider Kick-off Meeting, Geneva, Switzerland (2014).

[10] M-H. Wang et al., SLAC-PUB-16249. Submitted to the Journal of Instrumentation (2015). 